\title{
El uso de la secuencia didáctica en la Educación Superior
}

\author{
The Use of Didactic Sequence Strategy in Higher Education
}

\author{
Jéssica Araya-Ramírez ${ }^{1}$ \\ Escuela de Formación Docente \\ Universidad de Costa Rica \\ San José, Costa Rica \\ jesaraya30@yahoo.es
}

Recibido: 26 octubre $2012 \quad$ Aceptado: 13 marzo $2014 \quad$ Corregido: 25 marzo 2014

Resumen: Este artículo tiene como objetivo presentar los resultados de la sistematización de una experiencia pedagógica suscitada en la aplicación de la estrategia de aprendizaje denominada: "la secuencia didáctica (SD)", para desarrollar una unidad temática del curso FD-1027 Didáctica de la lectoescritura, el cual forma parte del plan de estudio de la Carrera de Educación Primaria de la Universidad de Costa Rica, con el propósito de mejorar los procesos de aprendizaje, sobre los principios didácticos, en relación las etapas de la composición de los textos en la etapa escolar. La ejecución de dicha innovación, se realizó desde el enfoque cualitativo y la investigación- acción, ya que interesaba describir, interpretar y comprender un problema, para reflexionar sobre el mismo y darle solución. En este sentido, se establecieron tres fases de trabajo en las que participaron 27 estudiantes. De acuerdo con los resultados se puede concluir que la estrategia permitió desarrollar de una manera lógica la unidad temática de las etapas de composición de la expresión escrita y se visualizó en el estudiantado un mayor empoderamiento de los principios didácticos, en la aplicación de la investigación en el área de la producción textual. Asimismo, es importante señalar que la implementación de la innovación formó parte de las actividades curriculares desarrolladas en el curso de Didáctica Universitaria, en el I semestre del 2012, para enriquecer los procesos de aprendizaje que fomenta el personal docente en las diversas carreras de la universidad.

Palabras claves: secuencia, didáctica, escritura, lectura, aprendizaje y enseñanza.

Abstract: This article aims to present the results of a systematic raised pedagogical experience in the application of the learning strategy called the didactic sequence (DS) to develop a thematic course unit in the FD-1027 Teaching Literacy course, which is part of the curriculum of the Primary Education School of the University of Costa Rica. The study's purpose was to improve the learning processes on didactic principles regarding the stages of composition of texts in the school years. The innovation implemented was an action-research which follows the qualitative approach, due to the interest of describing, interpreting and understanding a problem to then reflect upon it and find its appropriate solutions. In this regard, three phases of work were established with the participation of 27 students. According to the results, it can be concluded that the strategy allowed to develop the thematic unity of the stages of composition of writing in a logical way; and also, that it provided students' application of

Máster en lingüística de la Universidad de Costa Rica. Profesora de la Escuela de Formación Docente de la Universidad de Costa Rica, en el área de didáctica de la lengua española en la escuela primaria. Investigadora del Instituto de Investigaciones en Educación (INIE). 
research skills in textual production, which evidently generates a greater empowerment of educational principles. It is important to note that the implementation of this innovation was part of the curricular activities in the University Teaching course, in the first half of 2012, to enrich the learning process of university professors from different colleges on campus

Keywords: didactic sequence, writing, reading, learning, teaching.

\section{Introducción}

En el curso FD-0127 Didáctica de la lectoescritura, el cual forma parte de la estructura del plan de estudio de la Carrera de Bachillerato y Licenciatura en Educación Primaria de la Universidad de Costa Rica (Sede Rodrigo Facio), se desarrolla una unidad temática en relación con la composición de los textos en la etapa escolar. En este sentido, los y las estudiantes, deben lograr un dominio de los aspectos teóricos y metodológicos sobre los principios de la producción escrita a nivel escolar, para posteriormente proyectarlos en la práctica, en este caso, la implementación de una investigación en la expresión escrita con escolares de escuelas públicas.

Según los objetivos específicos planteados en el programa del curso, la unidad temática que se desarrolla en relación con la composición escrita contempla los siguientes aspectos:

1. Etapas de la composición de los textos escritos: planificación, textualización y revisión.

2. Elementos valorativos de la composición escrita: normativa (ortografía y variedad léxica), cohesión (puntuación, conectores y uso de sinónimos) y coherencia (estructura de las ideas de un texto en inicio, desarrollo y cierre)

Particularmente, como parte de la experiencia lograda, al desarrollar este curso durante algunos años, se ha visualizado cierta dificultad por parte de los y las estudiantes de la carrera, para integrar los aspectos teóricos y los principios metodológicos de la producción textual y su aplicación en la práctica.

Por lo tanto, de acuerdo con la carencia persistente en el desarrollo del curso, se implementó la estrategia de aprendizaje denominada: "la secuencia didáctica", la cual se seleccionó al permitir a corto plazo la sistematización según los objetivos de enseñanza establecidos, mejorar los procesos de aprendizaje de un área específica.

En este sentido, la estrategia didáctica utilizada contempla una serie de pasos que logran primeramente el desarrollo y la consolidación de las habilidades y los conocimientos teóricos necesarios, para posteriormente ejecutar una tarea específica, es decir, permite una integración adecuada entre teoría y práctica.

Al respecto, Camps citado por Vilá i Santasusana, Ballesteros, Castellá, Cros, Grau y Palou (2005, pp. 117-121) develan algunas razones que fundamentan los alcances y bondades de la secuencia didáctica (SD):

- Contempla el aprendizaje como un proceso más que un producto, en el que importa la adquisición y el desarrollo de competencias. 
- La secuencia didáctica da importancia a la evaluación formativa, la cual no gira en torno a un resultado final, sino más bien a un proceso de aprendizaje que pretende vincular habilidades y estrategias para lograr una meta.

- Permite determinar los conocimientos y habilidades necesarias para ejecutar una actividad.

- Vincula la autoreflexión y autoevaluación como parte del proceso de evaluación o valoración del propio aprendizaje.

Por lo tanto, de acuerdo con lo expuesto anteriormente, la aplicación de la estrategia mencionada, promueve una guía y una mediación que permiten valorar la adquisición de habilidades y conocimientos por parte de los y las estudiantes.

$\mathrm{Al}$ respecto, considerando las posibilidades de la aplicación de la estrategia didáctica, es que se plantearon como objetivos los siguientes:

- Aplicar la estrategia de la secuencia didáctica en el curso FD-1027 Didáctica de la lectoescritura, para desarrollar la unidad temática de las etapas de composición de la producción escrita.

- Analizar los alcances de la implementación de la estrategia de la secuencia didáctica en el curso FD-1027 Didáctica de la lectoescritura, para desarrollar la unidad temática de las etapas de composición de la producción escrita.

Asimismo, en cuanto al objetivo de este artículo, se contempló plasmar la estructuración de una sistematización, que permitiera no sólo conocer la descripción de la estrategia didáctica utilizada, sino también los logros obtenidos, con el propósito de demostrar al profesorado universitario la viabilidad de la implementación de la estrategia en cualquier temática, considerando de antemano la contextualización de esta, de acuerdo con la naturaleza de la disciplina.

Es importante señalar que para registrar la información se trabajó un registro de notas crudas, las cuales serán sistematizadas de acuerdo con las tres fases de aplicación de la estrategia. En este sentido, se considerará la conceptualización que formula Selener, Zapata y Purdy (1999; p.3) sobre el término "sistematización", el cual se refiere a un proceso continuo de reflexión participativa acerca de los procesos y resultados de un proyecto y es efectuado por los facilitadores y participantes del mismo.

En este caso, la valoración de la aplicación de la estrategia se hizo en dos vías, primeramente en cuanto a la reflexión del profesor que medió el proceso de aprendizaje y posteriormente, los y las estudiantes participantes del curso.

\section{Fundamentación teórica}

A continuación se detallan la conceptualización, los principios y características principales que contempla la estrategia de la secuencia didáctica: 
La secuencia didáctica es una propuesta metodológica que se deriva del "trabajo por tareas", el cual es un enfoque basado sobre el método por proyectos. Por lo que esta permite la programación sistematizada de contenidos conceptuales y procedimentales. En este sentido, tal como lo señala Vilá i Santasusana, Ballesteros, Castellá, Cros, Grau y Palou (2005; p.119), la secuencia didáctica es conceptualizada de la siguiente forma:

Consiste en pequeños ciclos de enseñanza y aprendizaje formados por un conjunto de actividades articuladas y orientadas a una finalidad... A su vez pretende articular de forma explícita los objetivos, los contenidos y las actividades en un proyecto de trabajo.

Es importante aclarar, que aunque la secuencia didáctica principalmente se contempla como una alternativa pedagógica. para el desarrollo de la didáctica de la lengua en general, puede ser aplicada en diversos ámbitos educativos y en el desarrollo de cualquier temática y disciplina.

A continuación se esbozan las siguientes características más importantes de esta estrategia, las cuales fundamentan lo anterior:

1. Pequeño ciclo de enseñanza y aprendizaje orientado a una finalidad y articulado en forma de secuencia temporal.

2. Los objetivos son concretos, limitados y compartidos con el alumnado.

3. Los procedimientos se centran en la reflexión sobre el uso y la aplicabilidad de los contenidos.

4. Incorpora las cuatro habilidades lingüísticas (hablar, escuchar, leer y escribir).

5. Las actividades se planifican desde una perspectiva bilateral: la función del emisor y la de los receptores.

6. Integra los distintos tipos de evaluación: inicial, formativa y sumativa. La evaluación formativa cobra una relevancia especial.

7. Prevé el uso de parrillas o pautas de observación y valoración en el proceso y los resultados.

8. Tiene una duración de 6 a 10 horas de clase. (Martín, 2009, p.120)

Dichas características en general, promueven un proceso de aprendizaje planificado de acuerdo con objetivos y contenidos delimitados, los cuales al ser implementados en la práctica, permiten determinar el grado de asimilación de los mismos y la funcionalidad en el campo de trabajo. Además, la evaluación del proceso valora primeramente los conocimientos previos de los sujetos, con el objetivo de enlazar los nuevos conceptos para posteriormente visualizar el manejo y la producción que realizan estos de la temática en estudio, lo cual genera una autoevaluación y autoregulación, es decir una reflexión metacognitiva del proceso de aprendizaje alcanzado.

Para lograr lo anterior, la secuencia didáctica contempla una estructura concisa y delimitada, en este sentido, Camps, citada por Vilá i Santasusana, Ballesteros, Castellá, Cros, Grau y Palou (2005; p.122), plantea el siguiente modelo: 


\section{Modelo de la secuencia didáctica}
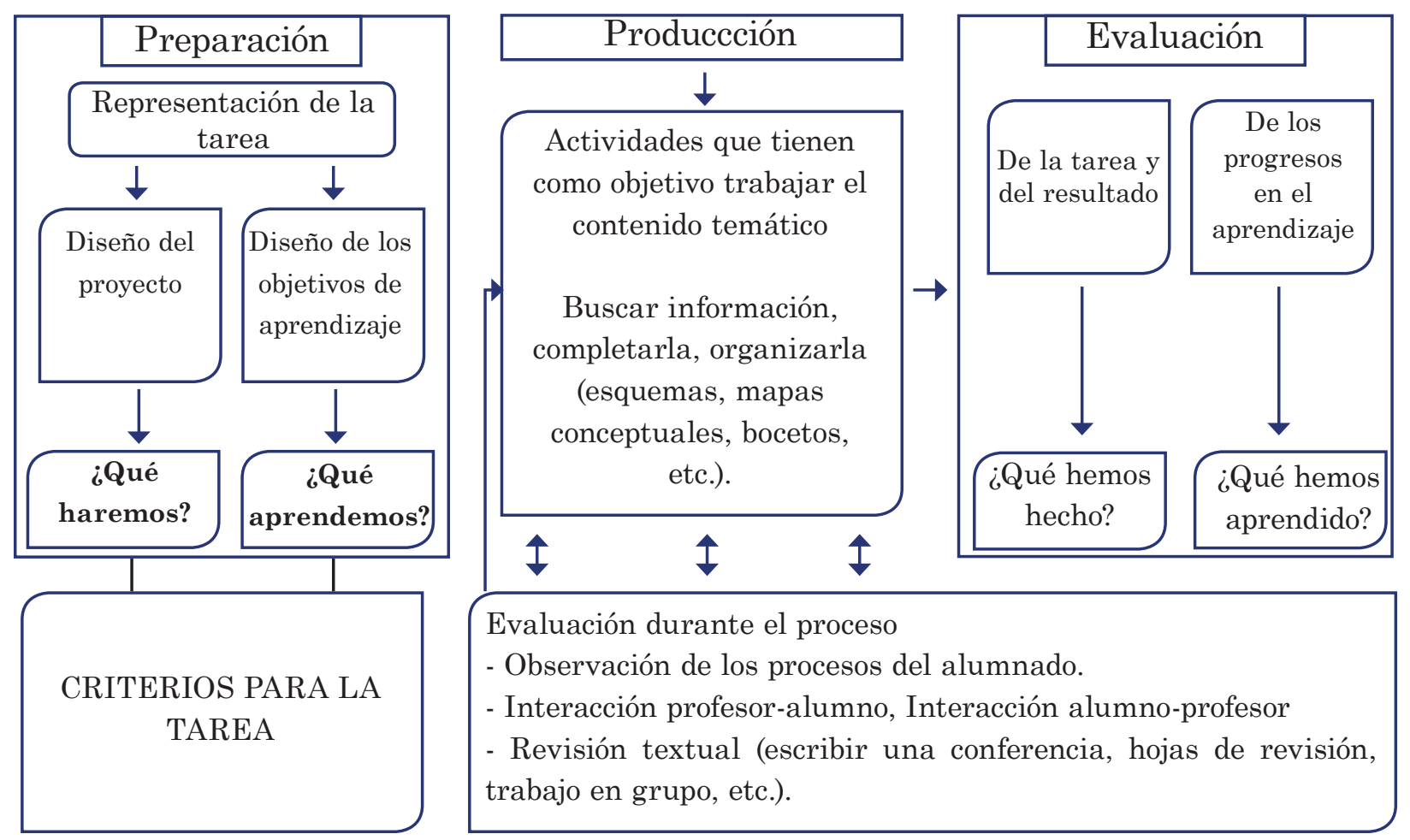

Figura 1. Adaptación del modelo de la secuencia didáctica

Fuente: Adaptación del modelo. Camps, citado por Vilá i Santasusana, Ballesteros, Castellá, Cros, Grau y Palou (2005; p. 122)

Tal como se aprecia en el gráfico anterior, la secuencia didáctica desarrolla tres etapas básicas:

- la preparación, la cual permite la planificación de la tarea y de lo qué se espera lograr, en este caso en conjunto docente y estudiante planifican el aprendizaje por lograr. Por lo tanto, se establecen, comprenden y asimilan los insumos necesarios para alcanzar los objetivos, ya sean los contenidos y procedimientos, a través de diversas estrategias.

- la producción, permite al sujeto la integración de la teoría y la práctica, pues deberá emplear los conocimientos construidos en las prácticas u otras actividades.

- y por último la evaluación, permite la sistematización de la experiencia y la autoevaluación de los logros alcanzados. Esta fase es sumamente valiosa, ya que permite determinar las debilidades y fortalezas suscitadas en el proceso de aprendizaje. 
Debido a lo anterior, la secuencia didáctica, como una alternativa en la adquisición y desarrollo de las competencias, induce al establecimiento de pautas claras acerca de la construcción del conocimiento y la integración de la teoría y la práctica, ya que posibilita que el docente gradúe los objetivos y contenidos de una unidad temática de forma sistemática.

Al implementar este tipo de estrategia se enfatiza el proceso de la adquisición de habilidades y conocimientos, por lo tanto, aduce más a la evaluación formativa y no tanto a la sumativa. Además, el error se visualiza como parte del proceso de aprendizaje y establece la reflexión de lo aprendido de una forma constante en la ejecución de las tres etapas de la estrategia.

Particularmente en la implementación de esta estrategia como innovación en el curso FD-1027 Didáctica de la lectoescritura, se delimitó la temática de la composición escrita en relación con las tres etapas de la producción textual, de acuerdo con el modelo de Flower y Hayes (1981) citado por Calsamiglia y Tusón (2008, p.70):

- Planificación: Se nutre de la memoria y del contexto pragmático e incluye la definición de objetivos, tanto los que se refieren a los procedimientos como a los contenidos, la generación de ideas y su organización.

- Textualización: Traduce los contenidos mentales en elementos de la lengua, con lo que genera decisiones a nivel del léxico-semántico, morfosintáctico y ortográfico.

- Revisión: Implica operaciones retroactivas de lectura que van evaluando los resultados de la textualización y de la acomodación a los objetivos iniciales.

De acuerdo con los autores, el dominio y la fundamentación teórica de dichas etapas por parte del personal docente, son necesarias para guiar el proceso de producción escrita, en este caso a nivel escolar, por lo que las características que plantea la estrategia pedagógica seleccionada, le permitirá a la población estudiantil del curso, en primer término, la apropiación de contenidos y procedimientos metodológicos de la temática y su respectiva aplicación en la práctica.

\section{Procedimiento metodológico}

Como se indicó la secuencia didáctica fue aplicada en el curso FD-1027 Didáctica de la lectoescritura para educación primaria, en este caso participaron 27 estudiantes matriculados en el mismo, compuesto por 5 hombres y 22 mujeres. El curso se ubica en el segundo año de la carrera y tiene como requisito el curso FD-1014 Artes del Idioma, en el cual se aborda en forma general el aprendizaje de las habilidades lingüísticas en la etapa escolar, asimismo, el curso en el cual se implementó la innovación, aborda las distintas metodologías para iniciar el proceso inicial- formal de la lectoescritura, así como la comprensión lectora y la producción escrita.

Considerando la dificultad que los y las estudiantes de la carrera comúnmente enfrentan para relacionar la teoría y la práctica, en relación con la mediación pedagógica sobre la composición de textos escritos en el nivel escolar, es que se propuso implementar 
una innovación que permitiera concretizar los principios didácticos de la producción textual en el campo de trabajo con escolares. Por lo que, visualizando las bondades de la estrategia de la secuencia didáctica, particularmente, la posibilidad de operacionalizar las habilidades y conocimientos requeridos para concretar o lograr los objetivos de aprendizaje, se consideró que esta se abordaría desde un enfoque cualitativo, ya que como señalan Rodríguez, Gil y Eduardo (2006; p.16) este presume el dinámico intercambio entre la teoría, los conceptos y los datos.

En este caso, se persigue no sólo relacionar la teoría con la práctica, sino también describirla, interpretarla y comprenderla desde un determinado contexto o fenómeno.

A su vez, al considerarse que la aplicación de la innovación se realizaría desde un marco de la reflexión misma del problema suscitado, es que la estrategia se plantea desde la investigación-acción, pues el propósito fundamental de la aplicación de la innovación es mejorar la relación entre teoría y práctica que los y las estudiantes universitarias como parte de sus habilidades profesionales deben desarrollar.

Tal como indica Zuber-Skerrit (1992) señalado por Latorre (2004, p.28) la investigaciónacción presenta las siguientes características:

- Los sujetos reflexionan y mejoran su propia práctica y su situación.

- Se vincula con rigor la reflexión y la acción.

- Se hace pública la experiencia no sólo a otros participantes, sino también a otras personas interesadas y preocupadas en la situación y el trabajo

Por otro lado, considerando las fases de la estrategia didáctica y la temática de la composición de los textos escritos, esta se organizó de la siguiente manera:

1. Fase de preparación: Se establecieron los conocimientos y habilidades necesarias para la mediación pedagógica que debe asignársele a la composición escrita producida por los y las escolares.

2. Fase de producción: Una vez consolidados y construidos los conocimientos teóricos sobre la producción escrita, se analizaron varios textos de niños y niñas escolares, lo cual resultó ser un entrenamiento previo al trabajo de campo de la investigación posterior que se desarrolló en las aulas escolares.

3. Fase de evaluación: Los y las estudiantes plantearon observaciones y reflexiones acerca de la integración de la teoría y la práctica. En este sentido, se realizaron apreciaciones críticas acerca de lo que plantean los teóricos en relación con el tema de la composición escrita y su aplicación en la práctica.

Para la aplicación de las tres fases se dispusieron de 10 horas de trabajo, es decir dos y media sesiones a lo largo del semestre.

En relación con el desempeño de roles e interacción de los involucrados en el proceso de aprendizaje, lo cual es una característica importante en la aplicación de la secuencia didáctica, se estableció lo siguiente: 
- Sobre el rol del docente: Se gestó un papel de "guía" o "mediador o mediadora" en la integración de conocimientos previos y otras experiencias del estudiantado en la fundamentación teórica de la composición de los textos, así como los elementos para valorar las redacciones, en este caso la normativa (ortografía y variedad léxica), la cohesión (uso de nexos, sinónimos y orden de los elementos de la frase) y la coherencia (organización de las ideas en un inicio, desarrollo y cierre).

Para ello se hizo uso de videos, diapositivas, demostraciones y materiales como hojas, marcadores, tijeras, entre otros.

- Sobre el rol del estudiantado: La población estudiantil se involucró en forma participativa a través de sus propias experiencias y conocimientos previos obtenidos en otros cursos en relación con la temática. Además, realizaron diversas lecturas de autores sobre el tratamiento de la composición escrita producida por los escolares. Por último, ejecutaron un trabajo de campo en una escuela pública sobre esta temática.

Tabla 1.

Distribución de las sesiones de trabajo para aplicar la secuencia didáctica

\begin{tabular}{|c|c|}
\hline $\begin{array}{c}\text { Preparación/planificación } \\
\text { Primera sesión } \\
\text { (4 horas) }\end{array}$ & $\begin{array}{c}\text { Producción } \\
\text { Segunda sesión } \\
\text { (4 horas) }\end{array}$ \\
\hline $\begin{array}{l}\text { - Los estudiantes fueron } \\
\text { informados acerca de la } \\
\text { estrategia que se implementaría } \\
\text { para desarrollar la unidad } \\
\text { temática del curso denominada: } \\
\text { La composición escrita en la } \\
\text { etapa escolar. En este caso } \\
\text { se analizaron las fases que } \\
\text { conforman la secuencia didáctica. } \\
\text { Con anterioridad los estudiantes } \\
\text { leyeron dos capítulos del libro: } \\
\text { Enseñar lengua (Cassany, Luna } \\
\text { y Sanz, 2008) sobre la temática a } \\
\text { desarrollar y a partir de la lectura } \\
\text { realizada y los conocimientos } \\
\text { previos de los estudiantes sobre } \\
\text { el tema, se construyó un bosquejo } \\
\text { de ideas en la pizarra sobre las } \\
\text { etapas de la composición de los } \\
\text { textos. En este caso, se analizó la } \\
\text { mediación pedagógica necesaria } \\
\text { en cada una de las etapas de la } \\
\text { composición escrita (planificación, } \\
\text { desarrollo y revisión). }\end{array}$ & $\begin{array}{l}\text { - Con el objetivo de incorporar los contenidos } \\
\text { analizados en la sesión anterior y de esta manera } \\
\text { integrar la teoría y la práctica, se diseñó un } \\
\text { entrenamiento sobre la planificación, textualización } \\
\text { y revisión de la composición escrita con el } \\
\text { estudiantado de la carrera. Lo cual les facilitaría } \\
\text { posteriormente, el trabajo de campo que debían } \\
\text { realizar estos con los niños y las niñas escolares. } \\
\text { - Como primera parte del entrenamiento se les } \\
\text { presentaron a los estudiantes varios estímulos } \\
\text { lingüísticos (los cuales son elementos importantes } \\
\text { que posibilitan la producción oral y escrita). } \\
\text { Considerando los intereses y la edad de los y } \\
\text { las estudiantes de la carrera, se utilizaron los } \\
\text { siguientes: } \\
\text { El video musical Peace and love de Fidel Nadal. } \\
\text { Nadal, F. (2012). Peace and love.[en línea] } \\
\text { Recuperado de: http:// www.youtube.com / } \\
\text { watch?v=6XwAdh9-9T4 } \\
\text { Elvideo musical "Nineteen", creado en el año 1985, } \\
\text { del cantante Paul Hardcastle, sobre la Guerra } \\
\text { de Vietnan Hardcastle, P. (1985). Nineteen [en } \\
\text { línea].Recuperado de: http://www.youtube.com/ } \\
\text { watch?v=JcFFEjq5Ap8 }\end{array}$ \\
\hline
\end{tabular}

\section{Evaluación \\ Tercera sesión \\ (dos horas)}

La fase de evaluación se realizó tomando como insumos: la experiencia del entrenamiento en clase $y$ el trabajo de campo realizado en una institución escolar.

En este caso se generaron dos preguntas que ellos debían responder en forma grupal:

¿Qué realizamos?

¿Qué hemos aprendido? 
- De acuerdo con el bosquejo de ideas realizado, se analizaron redacciones escolares en relación con los aspectos de normativa, cohesión y coherencia.

- Posteriormente se hizo una recapitulación de los contenidos y actividades anteriores.
- El video "Movimiento hippie".

Shiappa, J. (2012). Movimiento hippie. Recuperado de:http://www.youtube.com/ watch?v=bDf1lXetyns

- Posteriormente, los estudiantes se organizaron en cuatro grupos y de acuerdo con los estímulos lingüísticos presentados, elaboraron un pequeño texto con la implementación de las tres etapas de la composición de los escritos.

- Por lo tanto, en la planificación la población estudiantil desarrolló un esquema de ideas (a manera de borrador), en la etapa de textualización, realizaron la producción textual, en este caso consultaron el diccionario e internet, para profundizar en el tema si era necesario. Y por último en cuanto a la revisión del escrito, cada grupo hizo una relectura del texto y después cada uno aportó recomendaciones a los mismos.

- Luego, se hizo una recapitulación sobre el entrenamiento realizado en clase, en relación con la integración de los aportes teóricos de la composición escrita y la ejecución de la misma, lo cual igualmente retroalimentaría el trabajo de campo que los y las estudiantes de la carrera deberían realizar con los y las escolares dos semanas después.

Nota: Elaboración propia (2012)

A continuación se presenta una síntesis de las actividades que se programaron en la aplicación de la secuencia didáctica, las cuales podrían orientar la aplicación de la misma en otras áreas disciplinares:

\section{Análisis de los resultados del proceso desarrollado}

En relación con el análisis de los resultados, es importante señalar que la estrategia didáctica al ser desarrollada en tres sesiones de trabajo, no siempre contó con la presencia total de los y las estudiantes en cada uno de los períodos, por lo que en algunos momentos de la clase se tenían que dar explicaciones individualizadas a aquellos estudiantes que se encontraron ausentes, esto incidió en algunos momentos al sistematizar y analizar los resultados obtenidos. Los anterior es importante que los y las docentes lo tomen en cuenta al aplicar la estrategia, ya que la secuencia didáctica, al ser una serie de actividades secuenciadas para lograr un objetivo, requiere que el estudiantado participante se encuentre informado y comprenda la metodología de trabajo para que realmente pueda insertarse en el proceso de aprendizaje sin ningún vacío o desfase.

A continuación se analizan los resultados obtenidos en cada una de las fases de la secuencia didáctica: 


\subsection{En relación con la fase de planificación:}

Los y las estudiantes del curso mostraron apertura para participar en el trabajo propuesto, aunque en ocasiones algunos y algunas eran más consistentes e insistentes en sus intervenciones, por lo tanto, resultó ser un reto que todo el estudiantado compartieran sus conocimientos, experiencias y perspectivas sobre la temática que se analizó.

Particularmente, en otras ocasiones del curso, se les presentaba a los estudiantes una presentación de las ideas más importantes de la lectura, donde básicamente lo que se daba era una transmisión de ideas, lo cual estaba lejos realmente de la construcción del conocimiento por parte del estudiantado.

Asimismo, el grupo estudiantil logró satisfactoriamente la identificación de los conceptos fundamentales, que los autores proponen en relación con la composición de textos escritos, lo cual se evidenció con los criterios de valoración emitidos por el estudiantado al analizar las redacciones de escolares que se les suministraron el curso como una forma de entrenamiento.

Este tipo de entrenamiento permitió que los estudiantes lograran implementar los aspectos teóricos de la composición de los textos y en relación con los elementos de cohesión, normativa y coherencia, estos los operacionalizaron en la forma en que los niños y niñas escolares redactaban sus textos, por lo que mediante ejemplos concretos analizaron cada detalle de la producción textual de la población escolar.

En este sentido, la estrategia de la secuencia didáctica logró cumplir su cometido en el sentido de hilar fino la identificación y la comprensión por parte del alumnado, en cuanto a los fundamentos teóricos que respaldan la didáctica de la producción escrita en la etapa escolar y a su vez respaldan la praxis que debe ejecutar el futuro docente en relación con esta habilidad lingüística.

La secuencia didáctica tal como afirma González, Kaplan y Osua (2010, p.27-28) es una herramienta pedagógica en la construcción de competencias profesionales. En este caso, los autores agregan, que la estrategia en cuestión, permite al estudiantado ser generadores del conocimiento y de estrategias de aprendizaje, capaces de realizar la integración de saberes (saber, saber ser y saber hacer) con la experiencia cotidiana y que se constituyan en agentes de desarrollo social.

De acuerdo con lo anterior y la sistematización de la información que se registró en esta primera fase, fue evidente que el estudiantado logró relacionar los conceptos comprendidos sobre el tema, en las redacciones de los escolares que se analizaron en el curso, pues estos determinaban los errores más comunes en relación con la normativa, coherencia y cohesión que los niños y niñas fallan al producir textos escritos, desde una fundamentación teórica, vinculando y justificando cada situación particular.

De esta manera, el estudiantado de la carrera logró categorizar las debilidades y fortalezas de los textos analizados y de esa manera clasificaron los problemas detectados, agrupándolos por la carencia de conocimiento lingüístico por parte del escolar, con lo que se generó en la población estudiantil discusiones a nivel del léxico-semántico, morfosintáctico y ortográfico, en la construcción de los textos escritos por parte de los y las escolares. 


\subsection{En relación con la fase de producción:}

El identificar los intereses del grupo de estudiantes permitió que los estímulos lingüísticos elegidos tuvieran gran aceptación entre ellos, lo cual generó la elaboración fluida de los textos mediante la implementación de las tres etapas de la producción escrita. Esto resultó ser un entrenamiento muy funcional, ya que los estudiantes interiorizaron la puesta en práctica de los elementos teóricos que predican diversos autores sobre la construcción textual.

En relación con la autoevaluación de los escritos que realizaron los propios estudiantes surgieron dudas y comentarios, lo cual permitió generar una discusión y debate sobre la integración de la teoría y la práctica.

En esta actividad de la secuencia didáctica, el estudiantado reflexionó sobre sus propias dificultades al iniciar con un proceso de producción textual, en este sentido, su metacognición, le permitió iniciar la escritura de un texto, pero al enfrentar la fundamentación teórica relacionada con este proceso textual, debió modificar e integrar elementos primordiales para que su escrito cumpliera a cabalidad con las características de un texto redactado de manera correcta, el cual posteriormente le servirá como un texto modelo para mediar el aprendizaje de la composición escrita de la población escolar.

Particularmente, en esta segunda fase de la secuencia didáctica fue notable como los y las estudiantes integraron la práctica con la teoría, es decir, la experiencia o la vivencia de textualizar junto a sus compañeros y compañeras y discutir de manera paralela los preceptos teóricos que didácticamente aportan a esta actividad.

Al respecto Obaya y Ponce (2007, p. 19) se refiere a los aportes de de la secuencia didáctica en el proceso de aprendizaje de la siguiente manera:

La secuencia didáctica orienta y facilita el desarrollo práctico, la concebimos como una propuesta flexible que puede y debe, adaptarse a la realidad concreta a la que intenta servir, de manera que sea susceptible un cierto grado de estructuración del proceso de enseñanza aprendizaje con objeto de evitar la improvisación constante y la dispersión, mediante un proceso reflexivo en el que participan los estudiantes, los profesores, los contenidos de la asignatura y el contexto. Es además una buena herramienta que permite analizar e investigar la práctica educativa.

De acuerdo con ello, la secuencia didáctica permite organizar mejor los saberes para reconstruir y formular nuevas percepciones del conocimiento y cómo este permite fundamentar la propia práctica. Además, dentro de las bondades de esta estrategia, es evidente que la participación del estudiantado y la guía del docente promueven un proceso que insta al aprendizaje por descubrimiento, ya que se implementa la investigación como un principio didáctico para aprender, pues permite realizar comprobaciones, nuevas concepciones e hipótesis, que a su vez, favorecen la resolución de problemas prácticos.

Por lo tanto, uno de los mayores logros en esta fase de la secuencia didáctica, fue la reflexión del estudiantado acerca de la construcción del escrito realizado por ellos mismos, es 
decir las propias estrategias lingüísticas y el conocimiento previo que estos poseen para la elaboración de textos, los cuales fueron contrastados con la teoría, generando una actividad en el aula de búsqueda de respuestas, que permitió la reflexión-acción para la construcción del conocimiento científico y el desarrollo de las habilidades profesionales, particularmente como docentes en el campo de la didáctica de la lengua.

\subsection{En relación con la fase de evaluación:}

Esta fase se desarrolló en relación con las preguntas generadoras:

¿Qué realizamos?

¿Qué hemos aprendido?

Mediante dichas preguntas, los estudiantes lograron reflexionaron sobre su propio aprendizaje, por lo que según las apreciaciones recolectadas, se constató la necesidad de una planificación previa de las actividades de la composición escrita, pues estas nunca pueden ser improvisadas. A su vez la importancia de una consolida base teórica que de sustento a la mediación pedagógica que se hace al respecto.

En este sentido, el estudiantado tomó conciencia sobre la importancia de escoger del medio los elementos lingüísticos, para motivar a los escolares en cuanto a la producción de texto, asimismo la necesidad de que la mediación del docente en relación con el aprendizaje de la construcción de los escritos sea fundamentada con una base teórica, permite robustecer aún más el propio quehacer docente, lo cual le promueve al educador una mejor organización de mejor sus actividades y hacer los ajustes necesarios para que el proceso de aprendizaje se desarrolle de manera fluida y tomando en cuenta las características del contexto.

Además, en relación con el trabajo de campo en aulas escolares, los y las estudiantes lograron un mayor afianzamiento al desarrollar la investigación (en comparación con los años anteriores), pues se visualizó un mayor dominio del proceso de la producción textual, pues las actividades que seleccionaron en sus planes de aula para ser aplicados con los y las escolares, demostraba un hilo conductor de acuerdo con los objetivos planteados y las etapas de la composición textual, sin embargo, como en otras circunstancias, los mismos externaron lo difícil que fue trabajar la implementación de las etapas de la composición de los textos con los escolares, pues estos no presentan habilidades ni conocimientos al respecto.

Al respecto, sobre esta fase final de la estrategia didáctica, Feo Ronald (2010, p.230) afirma que:

(...) las actividades de cierre promueven la discusión y reflexión colectiva, buscan la forma que los estudiantes realicen de alguna manera, una representación que les ayude a recordar el proceso seguido. Estas estrategias deben orientar la atención de los estudiantes hacia la tarea, informando sobre lo correcto o incorrecto del resultado, promover de manera explícita la adquisición del aprendizaje, atribuyendo los resultados a causas percibidas como internas, modificables y controlables. 
En relación con lo anterior, es evidente que la secuencia didáctica permite una mejor organización de los objetivos de aprendizaje que el profesor o profesora plantea al estudiantado, además se modela y ejemplifica con situaciones concretas los conocimientos y las habilidades necesarias para que puedan desenvolverse adecuadamente en un contexto profesional determinado, en el que se espera como parte de sus competencias, la resolución a problemas de situaciones de su entorno inmediato.

Asimismo, los y las estudiantes de la carrera valoraron la puesta en práctica de la secuencia didáctica en el curso, los cuales de manera general manifestaron que la estrategia implementada les permitió sistematizar principalmente los conocimientos teóricos para enfrentar el trabajo de campo.

Dentro de las percepciones del estudiantado de la carrera sobre la aplicación de la misma se tienen las siguientes:

- Permite esclarecer dudas sobre los aspectos teóricos.

- Da espacios para integrar las experiencias y los conocimientos previos de los sujetos.

- Favorece la integración entre la teoría y la práctica

- El entrenamiento realizado permitió un mejor desarrollo del trabajo de campo (investigación en el área de la expresión escrita).

- La estrategia puede ser implementada en otros cursos de la carrera, así como en otros ambientes educativos.

Según estas apreciaciones, se visualiza que la aplicación de esta estrategia en el desarrollo del curso FD-1027 Didáctica de la lectoescritura, dista de los resultados que se obtienen del planteamiento de experiencias de aula más dirigidos al cúmulo de conocimientos sin conexión alguna y que en ocasiones sólo se memorizan pero no se operacionalizan en situaciones específicas del campo laboral.

Tal como señala Feo, Ronald (2010, p.235) la secuencia es en esencia un referente de los procedimientos de clase que el profesor de manera deliberada pretende desarrollar; sin embargo, es un procedimiento flexible que se adapta a las necesidades y expectativas del grupo.

Por ello, esta estrategia permite que el docente de manera sistemática y lógica organice los objetivos y contenidos de aprendizaje con un hilo conductor, que promueve no sólo la consecución de productos, sino también procesos que pueden ser retroalimentados sobre la marcha.

Asimismo, es importante señalar como aspecto a mejorar en próximas implementaciones de la estrategia didáctica, la importancia de generar y motivar más la participación del estudiantado, pues en ocasiones eran los mismos estudiantes los que participaban en las diversas actividades, además en algunos momentos, la población estudiantil tenía dificultades para utilizar el vocabulario técnico expresado por los autores analizados en clase, en relación con los ejemplos concretos de la expresión escrita desarrollada por escolares, lo cual debe seguirse reforzando de manera más habitual. 


\section{Conclusiones generales}

En relación con la experiencia desarrollada se presentan a continuación las consideraciones finales sobre la aplicación de la estrategia:

- De acuerdo con los objetivos planteados para la ejecución de la innovación se puede señalar que la estrategia de la secuencia didáctica, en el curso FD-1027 Didáctica de la lectoescritura, permitió planificar, desarrollar y evaluar de manera sistemática y lógica la unidad temática de las etapas de composición de la producción escrita. Esta realmente brindó claridad no sólo a la docente en relación sobre la puntualización concreta de los conocimientos lingüísticos y habilidades que el estudiantado debía adquirir, sino también visualizar los escollos que estos enfrentaron para comprender y construir el conocimiento en relación con esta temática y poder aplicarla en contextos reales de aprendizaje.

- Por otro lado, sobre el objetivo trazado en esta innovación, relacionado con el análisis de los alcances de la implementación de la estrategia en el curso, para desarrollar la unidad temática de las etapas de composición de la producción escrita, fue evidente que el estudiantado logró canalizar y experimentar por cuenta propia la relación entre teoría y práctica, lo cual le permitió desarrollar competencias profesionales como futuro mediador pedagógico en los procesos de aprendizaje centrados en la producción escrita de los escolares.

- Por ello, asimismo, los autores consideran que la secuencia didáctica es una estrategia que permite la retroalimentación entre la teoría y la práctica, lo cual permite generar nuevas teorías y nuevas prácticas para abordar el objeto de estudio en los procesos de aprendizaje.

- Por otro lado, es importante señalar que la implementación de la estrategia permitió la participación del estudiantado y el docente cumplió un rol de mediador pedagógico, porque se limita a guiar el proceso a partir de los conocimientos previos y experiencias de los y las estudiantes y no es un mero transmisor de información.

- A pesar de que la secuencia didáctica fue diseñada en un principio para apoyar los procesos de aprendizaje de la lengua materna, esta presenta características muy versátiles para ser aplicada en el desarrollo de cualquier temática y disciplina, por lo tanto, es una herramienta que puede apoyar los procesos académicos que se gestan en la universidad y en otros espacios educativos. Además, permite integrar una serie de recursos didácticos y no se limita al espacio físico del aula.

- La aplicación de una estrategia innovadora, como parte de las actividades del curso de Didáctica Universitaria, es una experiencia importante en el desarrollo profesional de cada docente de la Universidad de Costa Rica, pues es a través de la indagación y el descubrimiento de nuevas formas de cómo aprenden los adultos es que se perfecciona la labor docente. 


\section{Referencias bibliográficas}

Actis Sunchales, B. (2006). Taller de lengua. Santa Fe: Homo Sapiens.

Cassany i comas, D., Luna, M., Sanz G. (2008). Enseñar lengua. Barcelona:Graó.

Cassany i comas, D.(2005). Describir el escribir. Barcelona: Paidós.

Cassany i comas, D. (2007). Afilar el lapicero. Barcelona: ANAGRAMA.

Cassany i comas, D.(1999). Construir la escritura. Barcelona: Paidós:

Calsamiglia, H y Tusón, A.(2008) Las cosas del decir. Barcelona: Ariel

Feo, R. (2010). Orientaciones básicas para el diseño de estrategias didácticas. Tendencias Pedagógicas (16), 221-236.

González, M., Kaplan, J., Osua G., Osua, A.(julio-setiembre, 2010). La secuencia didáctica, herramienta pedagógica del modelo educativo. Enface, Udual, (46), 27-33.

Hardcastle, P.. (1985). Nineteen [video de Youtube]. Recuperado de http://www.youtube.com/ watch? =JcFFEjq5Ap8

Latorre, A. (2004) La investigación-acción. Barcelona: GRAÓ.

Lomas, C. (1999). Cómo enseñar a hacer cosas con palabras (Volumen I). Barcelona: Paidós.

Martín Vegas, R.(2009). Manual de Didáctica de la Lengua y literatura. Madrid: Síntesis.

Martínez, G., López, M., Gracida, Y. (2002). Del texto y sus contextos. México: Edere.

Mendoza Fillola, A. (2004). Didáctica de la lengua y la literatura. Madrid: Pearson.

Nadal, F. (2012). Peace and love.[video de Youtube]. Recuperado de: http:// www.youtube.com /watch?v=6XwAdh9-9T4

Obaya, A., y Ponce, R.(2007). La secuencia didáctica como herramienta del proceso de aprendizaje en el área Química-Biológica. Contacto-S, (63), 19-25-

Rodríguez, G., Gil, J., García, E. (2006). Métodos de investigación cualitativa. Madrid: Ediciones Alijibe. 
Selener D, Zapata L，Purdy C.(2009). Manual de sistematización participativa. Quito:Abya Yala.

Shiappa, J. (2012). Movimiento hippie. [video de Youtube] Recuperado de:http://www.youtube. $\mathrm{com} /$ watch? $\mathrm{v}=\mathrm{bDf} 11$ Xetyns

Vilá i Santasusana, M., Ballesteros, C,. Castellá, M,. Cros, A., Grau, M., y Palou J. (2005). El discurso oral formal. Barcelona: GRAÓ. 